\begin{tabular}{cc}
\hline & \\
& $\begin{array}{l}\text { International Journal of Case Reports } \\
\text { (ISSN:2572-8776) }\end{array}$
\end{tabular}

\title{
Mucin-producing papillary thyroidal carcinoma with unusual histology: A rare case report
}

\author{
Kimihide Kusafuka, D.D.S., Ph.D. ${ }^{1}$, Masaru Yamashita, M.D., Ph.D. ${ }^{2}$ \\ ${ }^{1}$ Department of Pathology, and ${ }^{2}$ Department of Otorhinolaryngology-Head and Neck Surgery, \\ Shizuoka General Hospital, Shizuoka, Japan
}

\section{ABSTRACT}

Background: Although mucinous carcinoma or mucoepidermoid carcinoma of the thyroid gland are an extremely rare but an established entity, papillary thyroidal carcinoma (PTC) with mucin production is also exceedingly rare. Case report: We reported one rare case of mucin producing PTC with unusual histology. The patient was 71 years-old Japanese man. The hemi-thyroidectomy was performed. Histologically, the tumor cells harbored the similar features with conventional PTC, but this case indicated extracellular and intracellular mucin. On the other hand, the tumor showed cystic-papillary or cribriform growth of the columnar tumor cells. Conclusions: We diagnosed it as mucin-producing PTC with columnar cell variant (CCV). We examined it immunohistochemically, and this case expressed TTF-1, thyroglobulin, and cytokeratin (CK)7, but was negative for HBME-1. We discuss on the differential diagnosis.

Keywords: papillary carcinoma, thyroid gland, mucins, FISH, columnar cell variant

Abbreviations: CCV, columnar cell variant; CDX-2, caudal family of homeobox transcription factor-2; CK, cytokeratin; EMA, epithelial membrane antigen; ETV6, translocation-Ets-leukaemia virus-6; FISH, fluorescence in situ hybridization; LMWK, low-molecular-weight keratin; MAML2, masterminded-like protein 2; MASC, mammary analogue-secretory carcinoma; MEC, mucoepidermoid carcinoma; PTC, papillary thyroidal carcinoma; TGB, thyroglobulin; TTF-1, thyroid transcription factor-1
*Correspondence to Author:

Kimihide Kusafuka, D.D.S., Ph.D.

Department of Pathology, Shizuoka General Hospital, 4-27-1 Kita-ando, Aoi-ku, Shizuoka city, Shizuoka 420-8527, JAPAN

How to cite this article:

Kimihide Kusafuka, Masaru Yamashita. Mucin-producing papillary thyroidal carcinoma with unusual histology: A rare case report. International Journal of Case Reports, 2020 4:131

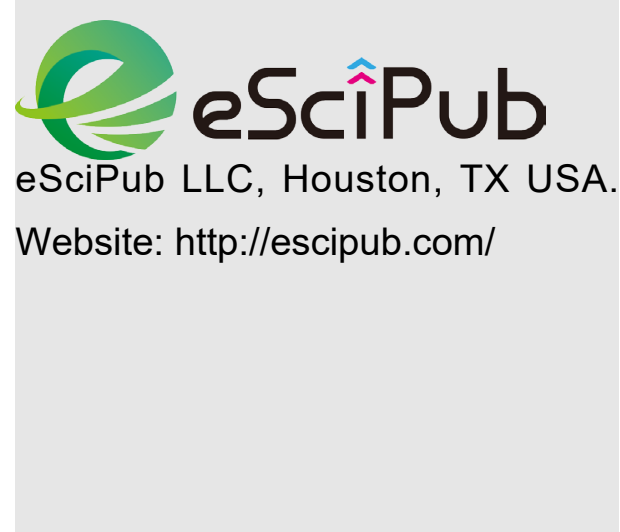




\section{Background}

Mucin-producing carcinoma of the thyroid gland is rare. Mucinous carcinoma ${ }^{1}$ and mucoepidermoid carcinoma (MEC) $)^{2}$ of the thyroid gland are extremely rare, but they are well-established entities. Although mucin production in several thyroidal carcinomas have been reported ${ }^{3,4,5}$, extensive mucin production in papillary thyroidal carcinoma (PTC) has not been reported. Generally, such carcinomas are characterized by pools of extracellular mucin, admixed with areas of typical thyroid carcinoma. This caused problems with differential diagnosis, especially with respect to the interpretation of mucinous deposits that occur in metastatic carcinomas. We report a case of mucin-producing PTC with an unusual histology.

\section{Case Report}

A 71-years-old Japanese man, underwent complete check-up. Computed tomography and magnetic resonance imaging showed a mass in the left lobe of the thyroid gland. After 16 months, he noticed a swollen lymph node in the left side of his neck. Subsequent fine needle aspiration cytology showed papillary carcinoma. Consequently, left hemi-thyroidectomy and the left neck dissection were performed. There was no recurrence nor metastasis after 1.5 years.

Grossly, a well-circumscribed yellowish mass, with partial hemorrhage, was observed in the left lobe of the thyroid gland. Histologically, the tumor was encapsulated by the dense fibrous tissue, showing papillary and/or cribriform growth pattern with extracellular and intracellular mucin (Figure 1A), which was positive for mucicarmine and Alcian blue stains (Figure 1B). The tumor cells showed nuclear grooves and cytoplasmic intranuclear inclusion bodies (Figure 1A: inset), which suggested that this tumor would be PTC. The metastatic lesions indicated cystic-papillary and/or fused-glandular structures, peripheral palisading, tubular spaces containing mucin, and extracellular mucin pool, which consisted of the columnar-shaped atypical cells with clear cytoplasm (Figure 1C). Markedly cellular anaplasia was not seen.

Immunohistochemistry was performed using a Leica BOND MAX automated immunostainer (Leica, Bannockburn, IL), Dako Autostainer Link 48 (DakoCytomation, Carpinteria, CA), or VENTANA BenchMark ULTRA automatic immunostainer (Roche Tissue Diagnosis, Oro Valley, AZ), according to the relevant manufacturer's protocol. We used Image J (National Institutes of Health, Bethesda, MD, USA) to estimate the percentage of $\mathrm{Ki}$-67-positive tumor cells. The tumor cells were positive for thyroid transcription factor (TTF)-1 (SP141; Biocare Medical Inc.; Pacheco, CA), thyroglobulin (TGB) (Dak-Tg6; DakoCytomation, Carpinteria, CA), cytokeratin (CK)7 (OV-TL-12/30; DakoCytomation), epithelial membrane antigen (EMA) (NCLEMA; Leica Biosystems; Nossloch. Germany), MUC1 (Ma695; Leica Biosystems) and MUC4 (8G7; Santa Cruz Biotechnology; Santa Cruz, CA) (Figure 1D, 1E, and 1F), but negative for anti-mesothelioma antibody (HBME-1; Nichirei Bioscience Inc. Tokyo, Japan), p63 (Dak-p63; DakoCytomation), calcitonin (polyclonal; Leica Biosystems), caudal family of homeobox 
transcription factor-2 [CDX-2] (Dak-CDX2; which suggested a wild type of TP53, whereas DakoCytomation), MUC2 (Ccp58; DakoCytoma- Ki-67 (MIB-1; DakoCytomation) labeling index tion), MUC5AC (45M1; Thermo Fisher Scientific, was 13.2\% (Figure 1G). We performed fluoresInc.; Waltham, MA), and MUC6 (CHL5; Abnova; cence in situ hybridization (FISH) for translocaTaipei, Taiwan). They were also partially positive tion-Ets-leukemia virus (ETV)6 and masterfor carcinoembryonic antigen (CEA) (II-7; Dako- minded-like protein (MAML)2, but both genes Cytomation), CK19 (RCK108; DakoCytomation), never showed breaking-apart (Figure 2A and and neural cell adhesion molecule (NCAM) (1B6; Leica Biosystems). The tumor cells were 2B). We finally diagnosed this case as "mucinproducing PTC, columnar cell variant (CCV)". focally positive for p53 (DO-7, DakoCytomation),

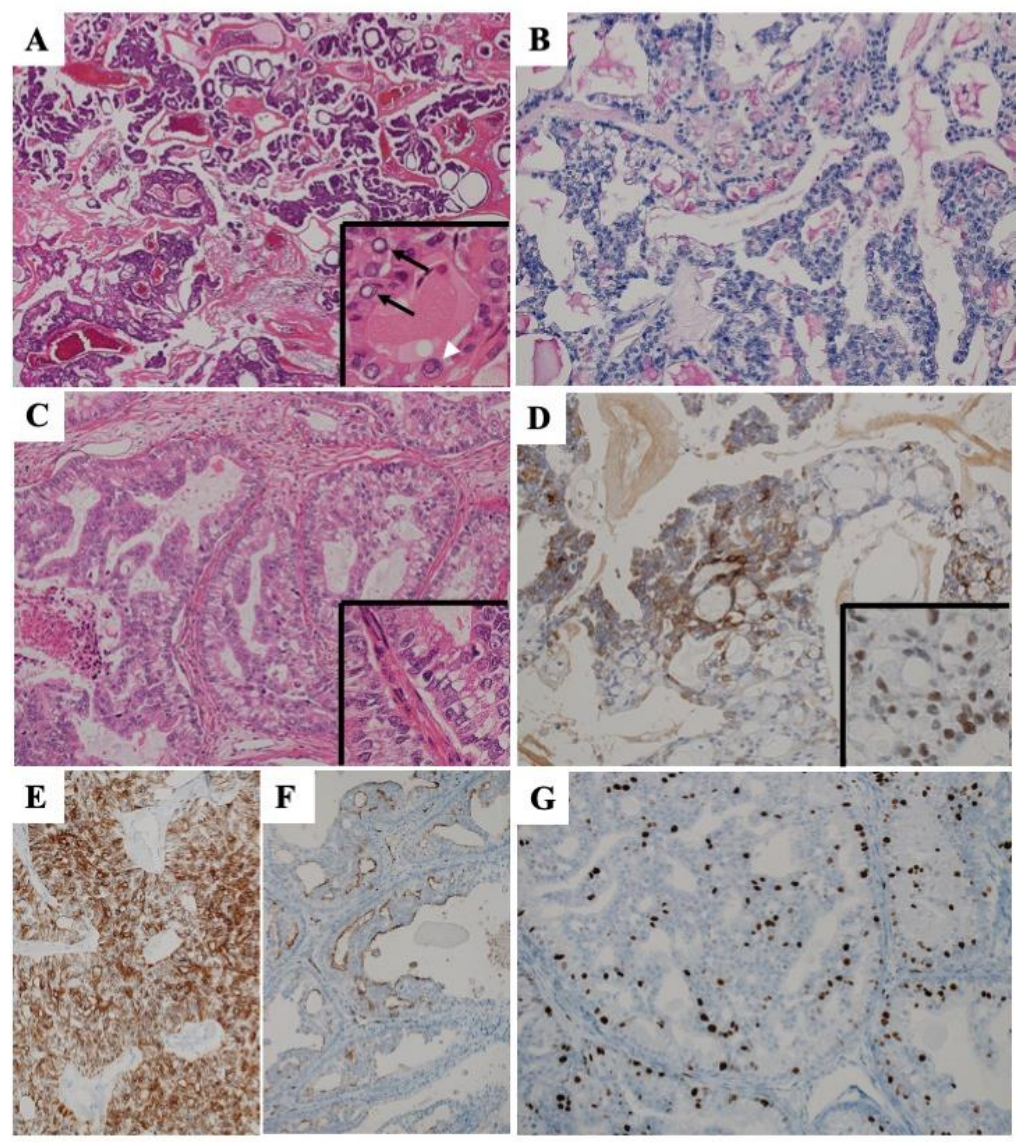

Figure 1: The primary tumor showed fused-glandular growth pattern with extracellular mucin (A; hematoxylin and eosin [H\&E] stain: Inset, intranuclear vacuoles [black arrows], pseudo-nuclear inclusion body [white arrowhead]). Extracellular and intracellular mucin was positive for mucicarmine stain (B; mucicarmine stain). The lymph node metastatic tumor showed cystic-papillary growth pattern of atypical cells with clear cytoplasm (C; H\&E stain: Inset, columnar tumor cells with clear cytoplasm and intranuclear vacuoles). The tumor cells were positive for thyroglobulin (D; immunostaining: Inset, they were also positive for TTF-1). The tumor cells were strongly and diffusely positive for CK7 (E; immunostaining), and they were positive for MUC4 at the luminal sides (F; immunostaining). The Ki-67 labelling index of the tumor was 13.2\% (G; immunostaining). 

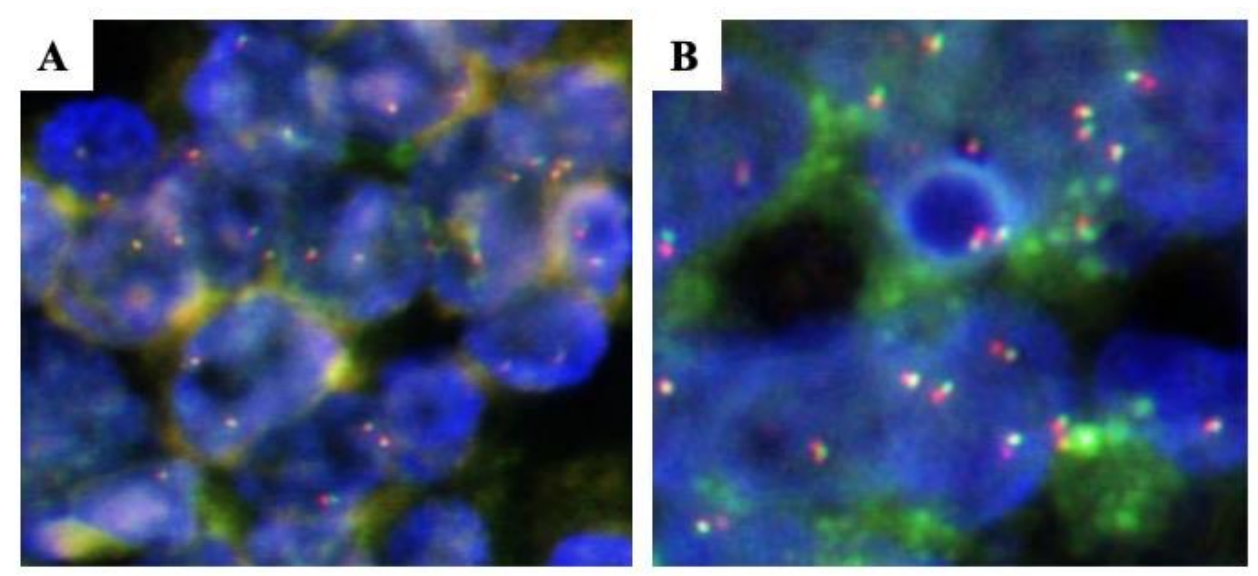

Figure 2: FISH analysis showed no breaking-apart of ETV6 (A) and MAML2 (B).

\section{Discussion}

PTC-CCV is a well-established entity as one of aggressive variants of $\mathrm{PTC}^{6}$, which is exceedingly rare. PTC-CCV cells usually harbor the clear and long cytoplasm and subnuclear vacuole, but they partially indicated the nuclear features, similar to conventional PTC. Mucin-producing PTC-CCV has not yet reported, although approximately $50 \%$ of PTC harbored mucin at the various levels, which was positive for mucicarmine and/or Alcian blue-periodic acid-Schiff stains.

The present case showed cystic-papillary and fused-glandular structures, and such a histology is unusual as PTC. Furthermore, the present PTC case produced extracellular and intracellular mucin and indicated a mucinous lake. This phenomenon is exceedingly rare in PTC. On the contrary, $42 \%$ of medullary carcinoma were reported to show the mucin production ${ }^{4}$, and as medullary carcinoma is positive for CEA and calcitonin, both immunostaining for calcitonin and Congo red stain should be performed for differential diagnosis. The cytological features of mucin-producing follicular carcinoma are shared by those of mucin-producing PTC, such as singular or cluster arrangement, high nucleus/cytoplasm ratio, nuclear grooves and evident nucleoli, and it was positive for TTF-1, NCAM, CK7 and CK19, but not for HBME-1 and p63 ${ }^{5}$. When diagnosing mucin-producing thyroidal tumors, such a variant of follicular carcinoma should be included in the differential diagnosis. Diffuse positivity for TTF-1 or CDX-2 suggests that the metastatic tumor is derived from lung cancers or colorectal cancers, respectively. The present case was positive for both TTF-1 and TGB. Therefore, the present case was not a metastatic tumor from the lung cancer. As the present case was negative for CDX-2, metastatic colorectal cancer was denied. Furthermore, PET should be performed due to the unclear origin of the metastatic tumors. The primary thyroidal mucinous carcinoma shows the cell clusters in the mucous lake, and immunohistochemically, it is positive for MUC2 and low-molecular-weightkeratin, adding partial positivity for TTF-1 and $\mathrm{TGB}^{1}$. If mucin hypersecretion is observed, 
mammary analogue-secretory carcinoma (MASC) $^{7}$ or mucoepidermoid carcinoma ${ }^{2}$ is also involved in the differential diagnosis. However, it is easy to differentially diagnose mucin-producing PTC from MASC and MEC using FISH analysis. Although mucin production is not relatively uncommon in thyroidal tumors, PTC with extensive mucin-production is extremely rare.

\section{Conflicts of interest}

The authors report no conflicts of interest. The authors alone are responsible for the content and writing of this article

Acknowledgement: We thank the staff of $\mathrm{Pa}$ thology Division, Shizuoka Cancer Center, Shizuoka, Japan, for immunohistochemistry and FISH.

\section{Ethical approval}

This study was approved by the institutional review board of Shizuoka General Center (SGHIRB\#2019007). All subjects signed informed consent forms to participate.

\section{Authors' contributions}

KK designed and drafted the manuscript. KK made the histopathological diagnosis and MY provided the clinical data. KK analyzed the immunohistochemical and FISH results. All of the authors have read and approved the final manuscript.

\section{References}

1. M. Sobrinho Simoes, J.M. Cameselle Teijerio, T. Kondo . "Mucinous carcinoma" Chapter 2: Tumours of the thyroid gland. In R.V. Lloyd, R.Y. Osamura, G. Kloppel, J. Rosai (Eds.) Pathology of the endocrine tumours World Health Organization classification, Lyon, France: IARC Press,
2017, p121

2. J.M. Cameselle Teijeiro, J. Albores Saaveeda, Z.W. Baloch, M. Sobrinho Simoes, B.M. Wenig. "Mucoepidermoid carcinoma" Chapter 2: Tumours of the thyroid gland. In R.V. Lloyd. R.Y. Osamura, G. Kloppel, J. Rosai (Eds.) Pathology of the endocrine tumours World Health Organization classification, Lyon, France: IARC Press, 2017, pp117118.

3. M.L. Mlynek, H.J. Richter, L.D. Leder. Mucin in carcinomas of the thyroid. Cancer 56 (1985) 2647-2650.

4. G.S. Zaatari, P.E. Saigo, A.G. Huvos. Mucin production in medullary carcinoma of the thyroid. Arch Pathol Lab Med 107 (1983) 70-74.

5. S. Squillaci , A. Pitino, C. Spairani , M. Ferrari , E. Carlon, M.F. Cosimi. Mucinous variant of follicular carcinoma of the thyroid gland: Case report and review of the literature. Int J Surg Pathol 24 (2016) 170-176.

6. M. Bongiovanni, M. Mermod, S. Canberk, C. Saglietti, G.P. Sykiotis, M. Pusztaszeri, M. Ragazzi, L. Mazzucchelli, L. Giovanella, S. Piana . Columnar cell variant of papillary thyroid carcinoma: cytomorphological characteristics of 11 cases with histological correlation and literature review. Cancer Cytopathol 125 (2017) 389-397.

7. S. Dogan, L. Wang, R.N. Ptashkin, R.R. Dawson, J.P. Shah, E.J. Sherman, R. Muchael Tuttle, J.A. Fagin, D.S. Klimstra, N. Katabi, R.A. Ghossein. Mammary analog secretory carcinoma of the thyroid gland: A primary thyroid adenocarcinoma harboring ETV6-NTRK3 fusion. Mod Pathol 29 (2016) 985-995. 